\title{
Geometric properties of the norm and basic sequences in Banach spaces
}

\section{Ivan Singer}

We introduce some generalizations of Kadec'-Klee norms and use them to study characteristics of subspaces of conjugate spaces and smoothness. We give some connections between such characteristics and basic sequences, which yield, in particular, sharpenings and simpler proofs of some known characterizations of reflexivity.

\section{1 .}

We shall say (following [7], where this notion and term have been introduced for spaces with separable conjugate spaces) that the norm of a Banach space $E$ is a (KK) norm (Kadec'-Klee norm) or, briefly, that $E$ is a (KK) space, if for every net $\left\{g_{d}\right\}_{d \in D} \subset E^{*}$ and every $g \in E^{*}$ such that $g_{d} \stackrel{\omega^{*}}{\longrightarrow} g,\left\|g_{d}\right\| \rightarrow\|g\|$, we have $\left\|g_{d}-g\right\| \rightarrow 0$. Here we introduce the following formally weaker notion: we shall say that $E$ is a sub-(KK) space if the above condition is satisfied for every $g \in P$, where $P$ is the set of functionals $g$ in $E^{*}$ which attain their norm (that is, for which there exists $x \in E$ with $\|x\|=1, g(x)=\|g\|)$. We shall also consider the countable versions of these notions, namely, we shall use the terms $\left(\mathrm{KK}_{\omega}\right)$ space or sub- $\left(\mathrm{KK}_{\omega}\right)$ space if one of the above conditions is satisfied for sequences $\left\{g_{n}\right\}$ instead of nets $\left\{g_{d}\right\}_{d \in D}$ (thus, $(K K) \Rightarrow\left(K_{\omega}\right) \Rightarrow \operatorname{sub}-\left(K_{\omega}\right)$ and $\left.(K K) \Rightarrow \operatorname{sub}-(K K) \Rightarrow \operatorname{sub}-\left(K_{\omega}\right)\right)$. In $\S 2$ we shall show that the results of [7] on characteristics of subspaces of the

Received 18 July 1975. 
conjugate space of a (KK) space with separable conjugate space remain valid for sub-(KK) spaces. In $\$ 3$ we shall show that either one of the sub-(KK) or sub- $\left(\mathrm{KK}_{\omega}\right)$ properties is the link between smoothness and Fréchet differentiability. In $\$ 4$ we shall give some complements to our results of [16] on non-smoothness of non-reflexive second conjugate spaces ${ }^{1}$. Finally, in $\$ 5$ we shall show that the converse of a connection between characteristics and basic sequences, observed in [16], is also true; in particular, this will yield sharpenings and simpler proofs of some known characterizations of reflexivity of Banach spaces. We shall consider only real Banach spaces, but the proofs can be easily extended to the complex case.

2.

We recall (see [6]) that the characteristic of a subspace $V$ (by subspace we shall always mean closed linear subspace) of a conjugate Banach space $E^{*}$ is the greatest number $r=r(V)$ such that the unit cell $S_{V}=\{f \in V \mid\|f\| \leq 1\}$ of $V$ is $\sigma\left(E^{*}, E\right)$-dense in the r-cell $r S_{E^{*}}=\left\{f \in E^{*} \mid\|f\| \leq r\right\}$ of $E^{*}$ (clearly, $\left.0 \leq r(V) \leq 1\right)$. In [7] it was proved that if $E$ is a (KK) space with separable conjugate space $E^{*}$, then for every proper subspace $V$ of $E^{*}$ we have $r(V)<I$. Now we shall prove that this result remains valid for arbitrary sub-(KK) spaces.

THEOREM 1. Let $E$ be a sub-(KK) space. Then for every proper subspace $V$ of $E^{*}$ we have $r(V)<1$.

Proof. Assume, a contrario, that $V$ is a (norm closed linear) subspace of $E^{*}$ with $V \neq E^{*}, r(V)=1$. Then there exists $g \in P \backslash V$ with $\|g\|=1$, where $P$ is the subset of $E^{*}$ occurring in the definition of sub-(KK) norms (indeed, by the Bishop-Phelps Theorem [4], $P$ is dense in $\left.E^{*}\right)$. Since $r(V)=1$, there exists a net $\left\{g_{d}\right\}_{d \in D} \subset V$ with $\left\|g_{d}\right\| \leq 1$ $(d \in D)$, such that $g_{d} \stackrel{w^{*}}{\longrightarrow} g$. Then $1=\|g\| \leq \underline{\lim }\left\|g_{d}\right\| \leq \overline{1 i m}\left\|g_{d}\right\| \leq 1$, whence $\left\|g_{d}\right\| \rightarrow\|g\|$. Consequently, since $E$ is sub-(KK), $\left\|g_{d}-g\right\| \rightarrow 0$, whence $g \in V$, a contradiction, which completes the proof.

1 The author takes this opportunity of stating that on page 410, line 14 , of $[16], " \Phi \neq 0 "$ should read $\Phi(f) \neq 0 "$. 
3.

Now we shall show that either one of the sub-(KK) or sub- $\left(K K K_{\omega}\right)$ properties is precisely the condition which must be added to smoothness (denoted by $(S)$ ), in order to obtain Fréchet differentiability of the norm of $E$ at every non-zero point (denoted by $(F)$ ).

THEOREM 2. For a Banach space $E$ the following statements are equivalent:

$1^{\circ}$. $E$ is $(F)$;

$2^{\circ}$. $E$ is $(S)$ and $s u b-(K K)$;

$3^{\circ}$. $E$ is $(S)$ and $s u b-\left(K K_{\omega}\right)$.

Proof. Clearly, $(F) \Rightarrow(S)$. Assume now that $E$ is $(F)$ and let $\left\{g_{d}\right\}_{d \in \mathcal{D}} \subset E^{*}, g \in P, g_{d} \stackrel{w^{*}}{\longrightarrow} g,\left\|g_{d}\right\| \rightarrow\|g\|$. Since $g \in P$, let $x \in E,\|x\|=1, g(x)=\|g\|$. Then, since $g_{d} \stackrel{\omega^{*}}{\longrightarrow} g$, for every $\varepsilon>0$ there exists $d_{0}=d_{0}(\varepsilon, x)$ such that $\left|g_{d}(x)-\|g\|\right|=\left|g_{d}(x)-g(x)\right|<\varepsilon$, whence $g_{d}(x)>\|g\|-\varepsilon$, whenever $d \geq d_{0}(\varepsilon, x)$. But, since $E$ is $(F)$, by a result of $\zeta_{m u l i a n}[19]$ we have

$$
\operatorname{diam} A_{x, \varepsilon,\|g\|} \rightarrow 0 \text { as } \varepsilon \ngtr 0 \text {, }
$$

where $A_{x, \varepsilon,\|g\|}=\left\{h \in E^{*} \mid\|h\| \leq I, h(x) \geq\|g\|-\varepsilon\right\}$. Hence, since $g_{d}, g \in A_{x, \varepsilon,\|g\|}$ for $d \geq d_{0}(\varepsilon, x)$, we obtain $\left\|g_{d}-g\right\| \rightarrow 0$, so $E$ is sub-(KK). Thus, $1^{\circ} \Rightarrow 2^{\circ}$.

The implication $2^{\circ} \Rightarrow 3^{\circ}$ is obvious.

Finally, assume that $E$ is both $(S)$ and sub- $\left(\mathrm{KK}_{\omega}\right)$ and let $x_{n}, x \in E,\left\|x_{n}\right\|=\|x\|=1, x_{n} \rightarrow x$. Then, by $(S)$, there are uniquely determined $f_{x_{n}}, f_{x} \in E^{*}$ with $\left\|f_{x_{n}}\right\|=\left\|f_{x}\right\|=1, f_{x_{n}}\left(x_{n}\right)=f_{x}(x)=1$, and they satisfy $f_{x_{n}} \stackrel{\omega^{*}}{\longrightarrow} f_{x}$, [9]. Hence, since $E$ is sub-(KK $\left.\mathrm{K}_{\omega}\right)$, we obtain $\left\|f_{x_{n}}-f_{x}\right\| \rightarrow 0$. Thus, the "support mapping" $x \rightarrow f_{x}$ is norm to 
norm continuous on $\sigma_{E}=\{x \in E \mid\|x\|=1\}$, whence $E$ is (F), [9]. Thus, $3^{\circ} \Rightarrow 1^{\circ}$, which completes the proof.

REMARK I. We recall that a Banach space $E$ is said to be (VS) (very smooth) if it is smooth and if the support mapping $x \rightarrow f_{x}$ is continuous on $\sigma_{E}=\{x \in E \mid\|x\|=1\}$ from the norm topology of $E$ to the $\sigma\left(E^{*}, E^{* *}\right)$ topology of $E^{*}$, or, what is equivalent [10], if the canonical image of $E$ in $E^{* *}$ is smooth as a subspace of $E^{* *}$ (that is, with respect to the functionals in $E^{* * *}$ ). Sullivan has proved ([20], Theorem 3 ) that if $E$ is (S) and (KK), then $E$ is (VS). Since (F) implies (VS) [20], [10], the implications $2^{\circ} \Rightarrow 1^{\circ}$, and $3^{\circ} \Rightarrow 1^{\circ}$ of Theorem 2 above are sharpenings of Sullivan's Theorem. The equivalence $1^{\circ} \Leftrightarrow 3^{\circ}$ can be also proved by using characterizations of $(F)$ in terms of "strongly smooth" points, and of (S), due to Smulian [18], [17] (see also [11]); in the particular case of reflexive spaces, essentially this latter equivalence has been obtained, with a different method, in [5]. In [14], Proof of Theorem 3, it has been shown, with the argument of the above proof of the implication $3^{\circ} \Rightarrow 1^{\circ}$, that $(S) \wedge\left(\mathrm{KK}_{\omega}\right) \Rightarrow(\mathrm{F})$.

REMARK 2. From Theorems 1 and 2 it follows that if $E$ is (F), then for every proper subspace $V$ of $E^{*}$ we have $r(V)<1$. This can be also proved as follows: by [20], [10], (F) implies (VS), and by [16], Theorem I, (VS) implies that every proper subspace $V \subset E^{*}$ has $r(V)<I$.

Problem 1. If $E$ is sub-(KK), or if $E$ is (F), does $E$ admit an equivalent $(\mathrm{KK})$-norm? What about the similar questions for sub- $\left(\mathrm{KK}_{\omega}\right)$, $\left(\mathrm{KK}_{\omega}\right)$ ?

4 .

In [16] we have proved some results on the open problem whether the second conjugate $E^{* *}$ of a non-reflexive Banach space $E$ is non-smooth, showing that for a large class of spaces this is indeed the case. Here we want to point out some more consequences of the results of [16] in the same direction.

THEOREM 3. If a Banach space $E$ contains a subspace isomorphic to 
$c_{0}$ or $l^{1}$, then $E^{* *}$ is not smooth.

Proof. The statement on $c_{0}$ was observed in [16]. On the other hand, if $E$ contains a subspace $G$ isomorphic to $l^{l}$, then dens $G<\operatorname{dens} G^{*}$, whence by [16], Corollary $1, G^{* *} \equiv G^{11} \subset E^{* *}$ is not smooth, so $E^{* *}$ is not smooth, which completes the proof.

COROLLARY 1. If $E$ is a non-reflexive subspace of a Banach space with an (uncountable or countable) unconditional basis, then $E^{* *}$ is not smooth.

Proof. By [2], $E$ satisfies the condition of Theorem 3 above.

More generally, the same conclusion also holds for non-reflexive subspaces of cyclic Banach spaces and of $\sigma$-complete and $\sigma$-order continuous Banach lattices ([21], Theorems 8 and 18).

5.

We recall that a sequence $\left\{x_{n}\right\}$ in a Banach space $E$ is called a basic sequence, if it is a basis of $\left[x_{n}\right]$, the closed linear subspace of $E$ spanned by $\left\{x_{n}\right\}$. A basic sequence $\left\{x_{n}\right\} \subset E$ is said to be

(a) asymptotically monotone, if $\underset{n \rightarrow \infty}{\lim }\left\|s_{n}\right\|=1$, where $s_{n}$ is the $n$-th partial sum operator $\sum_{i=1}^{\infty} \alpha_{i} x_{i} \rightarrow \sum_{i=1}^{n} \alpha_{i} x_{i}$ (defined on $\left.\left[x_{n}\right]\right)$

(b) shrinking, if $\lim _{n \rightarrow \infty}\left\|\left.f\right|_{\left[x_{i}\right]_{i=n}^{\infty}}\right\|=0$ for all $f \in E^{*}$.

In [16] we have observed that if $E$ contains an asymptotically monotone non-shrinking basic sequence, then $E$ has a subspace $F$ (namely, $\left.F=\left[x_{n}\right]\right)$ such that $F^{*}$ contains a proper subspace $V$ with $r(V)=1$. Now we shall prove that the converse is also true and we shall deduce some consequences; the techniques of the proof below, which is a natural extension of the techniques of Mazur (see [3].) and Gelbaum [8], may be of interest. for other applications too. 
We recall that if $G, Y$ are subspaces of a Banach space $E$ and $\sigma_{G}=\{x \in G \mid\|x\|=1\}$, the number

$$
(\widehat{G ; Y})=\operatorname{dist}\left(\sigma_{G}, Y\right)
$$

is called the inclination of $G$ to $Y$. In the proof of Lemma 1 below we shall use the fact that for a subspace $V$ of a conjugate space $E^{*}$ we have $r(V)=I$ if and only if $\|x\|=\sup |f(x)|$ for all $x \in E,[6]$. $f \in V$
$\|f\| \leq 1$

LEMMA 1. Let $E$ be a Banach space, $V$ a linear subspace of $E^{*}$ with $r(V)=1, G$ a finite-dimensional subspace of $E$ and $0<\varepsilon<1$. Then there exists a finite set of functionals $\left\{h_{1}, \ldots, h_{m}\right\} \subset V$ such that the finite codimensional subspace $Y=\prod_{i=1}^{m} \operatorname{ker} h_{i}$ of $E$ satisfies

$$
(\widehat{G ; Y})>1-\varepsilon \text {. }
$$

In particular, if $\operatorname{dim} G=1$, one can take $m=1, y=\operatorname{ker} h_{1}$.

Proof. Since $\operatorname{dim} G<\infty$, the set $\sigma_{G}=\{x \in G \mid\|x\|=1\}$ is compact and hence it has a finite $\varepsilon^{\prime}$-net $z_{1}, \ldots, z_{m}$, where $0<\varepsilon^{\prime}<\frac{\varepsilon}{2}$. Since $r(V)=1$, there exist $h_{1}, \ldots, h_{m} \in V$ such that $\left\|h_{i}\right\| \leq 1$, $\left|h_{i}\left(z_{i}\right)\right| \geq 1-\varepsilon^{\prime} \quad(i=1, \ldots, m)$. Then for every $x \in \sigma_{G}$ and $y \in Y=\bigcap_{i=1}^{m} \operatorname{ker} h_{i}$ we have, with a suitable $z_{i_{0}}$, $\|x+y\| \geq\left\|z_{i_{0}}+y\right\|-\left\|x-z_{i_{0}}\right\| \geq\left|h_{i_{0}}\left(z_{i_{0}}+y\right)\right|-\varepsilon^{\prime}=\left|h_{i_{0}}\left(z_{i_{0}}\right)\right|-\varepsilon^{\prime} \geq 1-2 \varepsilon^{\prime}$, whence $(\widehat{G ; Y}) \geq 1-2 \varepsilon^{\prime}>1-\varepsilon$. If $\operatorname{dim} G=1$, then $\sigma_{G}$ consists of two antipodal elements, say $\sigma_{G}=\left\{z_{1},-z_{1}\right\}$ and thus any $h_{1} \in V$ with $\left\|h_{1}\right\| \leq 1, \quad\left|h_{1}\left(z_{1}\right)\right| \geq 1-\varepsilon^{\prime}>1-\varepsilon$ works.

LEMMA 2 (Banach [1], Theortiu I, p. 122). Let E be a Banach space, $\Gamma$ a $w^{*}$-closed Zinear subspace of $E^{*}, f \in E^{*} \backslash \Gamma$ and $0<\varepsilon<\operatorname{dist}(f, \Gamma)$. Then there exists an element $x \in E$ such that

$$
h(x)=0 \quad(h \in \Gamma),
$$




$$
\begin{aligned}
f(x) & =1, \\
\|x\| & \leq \frac{1}{\operatorname{dist}(f, \Gamma)-\varepsilon} .
\end{aligned}
$$

THEOREM 4. A Banach space $E$ has a subspace $E_{0}$ such that $E_{0}^{*}$ contains a proper subspace $V$ with $r(V)=1$ if and only if $E$ contains an asymptotically monotone non-shrinking basic sequence.

Proof. As we mentioned already in the preceding, the sufficiency part was observed in [16].

Conversely, it is clearly enough to prove the necessity part under the assumption that $E^{*}$ contains a proper subspace $V$ with $r(V)=1$ (since an asymptotically monotone non-shrinking basic sequence $\left\{x_{n}\right\}$ in $E_{0} \subset E$ has the same properties in $E$ ).

Let $f \in E^{*} \backslash V, \quad 0<\varepsilon_{n}<\operatorname{dist}(f, V), \lim _{n \rightarrow \infty} \varepsilon_{n}=0$. Put $M=\frac{1}{\operatorname{dist}(f, V)-\varepsilon_{I}}$

Since $\|f\| \geq \operatorname{dist}(f, V)>\frac{1}{M}$, there exists $x_{1} \in E$ with $\left\|x_{1}\right\| \leq M$ such that $f\left(x_{1}\right)=I$. Then, by Lemma $I$ for $G_{1}=\left[x_{1}\right]$, there exists $h_{0} \in V$ such that $Y_{1}=\operatorname{ker} h_{0}$ satisfies $\left(\widehat{G_{1} ; Y_{1}}\right)>1-\varepsilon_{1}$. By Lemma 2 for $\Gamma_{1}=\left[h_{0}\right] \subset V \quad\left(\Gamma_{1}\right.$ is $w^{*}$-closed since $\left.\operatorname{dim} \Gamma_{1}<\infty\right)$ and for $f \in E^{*} \backslash V \subset E^{*} \backslash \Gamma_{1}$, there exists an element $x_{2} \in \operatorname{ker} h_{0}=Y_{1}$ with $f\left(x_{2}\right)=1,\left\|x_{2}\right\| \leq \frac{1}{\operatorname{dist}\left(f, \Gamma_{1}\right)-\varepsilon_{1}} \leq \frac{1}{\operatorname{dist}(f, V)-\varepsilon_{1}}=M$. Next, by Lemma 1 for $G_{2}=\left[x_{1}, x_{2}\right]$, there exists a finite-dimensional subspace $\Gamma_{2}=\left[h_{i}\right]_{i=0}^{m} \subset V$ with $\Gamma_{2} \supset\left[h_{0}\right]=\Gamma_{1}$, such that $Y_{2}=\bigcap_{i=0}^{m} \operatorname{ker} h_{i}$ satisfies $\left(\widehat{G_{2} ; Y_{2}}\right)>1-\varepsilon_{2}$. By Lemma 2 for $\Gamma_{2} \subset V$ and $f \in E^{*} \backslash \Gamma_{2}$, there exists an element $x_{3} \in Y_{2}$ with $f\left(x_{3}\right)=1,\left\|x_{3}\right\| \leq M$. Continuing in this way indefinitely, we obtain a sequence $\left\{x_{n}\right\} \subset E$ and a sequence of subspaces $\left\{y_{n}\right\}$ of $E$, such that 
(1) $\left(\overline{\left[x_{i}\right]_{i=1}^{n} ; Y_{n}}\right)>1-\varepsilon_{n}, 0 \neq x_{n+1} \in Y_{n}, Y_{n} \supset Y_{n+1}(n=1,2, \ldots)$,

$$
f\left(x_{n}\right)=1,\left\|x_{n}\right\| \leq M \quad(n=1,2, \ldots) .
$$

Then, by (1), $\left\{x_{n}\right\}$ is an asymptotically monotone basic sequence [3], [8] and, by (2), $\left\{x_{n}\right\}$ is non-shrinking (even "of type $P^{*}$ " in the sense of [15]), which completes the proof.

COROLLARY 2. If dens $E$ < dens $E^{*}$, or if $E$ contains a subspace $E_{0}$ isometric to a non-reflexive conjugate space $B^{*}$, then $E$ contains an asymptotically monotone non-shrinking basic sequence.

Proof. In both cases it is well known (see, for example, [16]) that $E$ satisfies the condition of Theorem 4 .

REMARK 3. The weaker result that every non-reflexive second conjugate space $B^{* *}$ contains an asymptotically monotone non-shrinking basic sequence, has been proved by Pełczyński [13], with a different method.

COROLLARY 3. If $E$ is a Banach space and $V$ is a proper subspace of $E^{*}$ with $r(V)>0$, then $E$ contains a non-shrinking basic sequence $\left\{x_{n}\right\}$ with partial sum operators $\left\{s_{n}\right\}$ satisfying $\overline{\lim }_{n \rightarrow \infty}\left\|s_{n}\right\| \leq \frac{1}{p(V)}$. Proof. As was observed by Dieudonné (see [6]), $\|x\| \|=\sup _{\substack{f \in V \\\|f\| \leq 1}}|f(x)|$

$(x \in E)$ is an equivalent norm on $E$, namely, $\|x\| \leq \leq\|x\| \leq \frac{1}{r(V)}\|x\|$ $(x \in E)$, and in this new norm we have ${ }^{2} \|_{\|\cdot\|}(V)=1$. Hence, by Theorem 4, $(E,|\|\cdot\||)$ has an asymptotically monotone non-shrinking basic sequence $\left\{x_{n}\right\}$, say, with $\left\|s_{n}\right\| \mid \leq 1+\varepsilon_{n}(n=1,2, \ldots)$, where $\varepsilon_{n}>0$, $\lim _{n \rightarrow \infty} \varepsilon_{n}=0$. But then

$$
\left\|s_{n}(x)\right\| \leq \frac{1}{r(V)}\left\|s_{n}(x)\right\| \leq \frac{1+\varepsilon_{n}}{r(V)}\|x\| \leq \frac{1+\varepsilon_{n}}{r(V)}\|x\| \quad(x \in E, n=1,2, \ldots),
$$

whence $\varlimsup_{n \rightarrow \infty}\left\|s_{n}\right\| \leq \frac{1}{r(V)}$, which completes the proof.

REMARK 4. It is well known and immediate that $E^{*}$ contains a proper 
subspace $V$ with $r(V)>0$ if and only if $E$ is non-reflexive. Thus the above results constitute a sharpening of the theorem of Pełczyński [12], [13] that every non-reflexive Banach space $E$ contains a nonshrinking bounded basic sequence $\left\{x_{n}\right\}$ such that $f\left(x_{n}\right) \geq 1$ $(n=1,2, \ldots)$ for some $f \in E^{*}$ (this result was first obtained for spaces with bases in [15]) and hence also of the classical Eberlein-Šmulian theorem that every non-reflexive space $E$ contains a bounded sequence which has no weakly convergent subsequence (indeed, if a basic sequence $\left\{x_{n}\right\}$ has a weakly convergent subsequence $\left\{x_{n_{k}}\right\}$, then clearly $\left.x_{n_{k}} \stackrel{w}{\longrightarrow} 0\right)$. Note that the above proof is considerably simpler than those of [12], [13], and it avoids the use of the second conjugate space $E^{* *}$.

\section{References}

[1] Stefan Banach, Théorie des opérations Linéaires (Monografje Matematyczne, 1. Z subwencji Funduszu Kultury Narodowej, Warszawa, 1932).

[2] C. Bessaga and A. Pełczyński, "A generalization of results of R.C. James concerning absolute bases in Banach spaces", Studia Math. 17 (1958), 165-174.

[3] Cz. Bessaga, A. Pełczyński, "Własności baz w przestrzeniach typu $B_{0}$ " [Properties of bases in spaces of type $B_{0}$ ], Prace Mat. 3 (1959), 123-142.

[4] E. Bishop and R.R. Phelps, "A proof that every Banach space is subreflexive", Bull. Amer. Math. Soc. 67 (1961), 97-98.

[5] D.F. Cudia, "Rotundity", Convexity, 93-97 (Proc. Sympos. Pure Math., 7. Amer. Math. Soc. Providence, Rhode Island, 1963).

[6] J. Dixmier, "Sur un théorème de Banach", Duke Math. J. 15 (1948), 1057-1071.

[7] Dick van Dulst and Ivan Singer, "On Kadec-Klee norms on Banach spaces", Studia Math. 54 (1975), 205-211. 
[8] Bernard R. Gelbaum, "Notes on Banach spaces and bases", An. Acad. Brasiz. Ci. 30 (1958), 29-36.

[9] J.R. Giles, "On a characterisation of differentiability of the norm of a normed linear space", J. Austral. Math. Soc. 12 (1971), $106-114$.

[10] J.R. Giles, "On smoothness of the Banach space embedding", Buzl. Austral. Math. Soc. 13 (1975), 69-74.

[11] Joram Lindenstrauss, "On operators which attain their norm", Israel J. Math. 1 (1963), 139-148.

[12] A. Pełczyński, "A note on the paper of I. Singer 'Basic sequences and reflexivity of Banach spaces'", Studia Math. 21 (1961-1962), $371-374$.

[13] A. Pełczyński, "A proof of Eberlein-Šmulian theorem by an application of basic sequences", Buzl. Acad. Polon. Sci. Sér. Sci. Math. Astronom. Phys. 12 (1964), 543-548.

[14] Guillermo Restrepo, "Differentiable norms", Bol. Soc. Mat. Mexicana (2) $10(1965), 47-55$.

[15] 1. Singer, "Basic sequences and reflexivity of Banach spaces", Studia Math. 21 (1961-1962), 351-369.

[16] I van Singer, "On the problem of non-smoothness of non-reflexive second conjugate spaces", Bull. Austral. Math. Soc. 12 (1975), $407-416$.

[17] В.Л. Шиульян [V.L. Smulian], "О некоторых геометричесних свойствах единичной сферы пространства типа (B) " [On some geometrica] properties of the unit sphere in the space of type (B) ], Mat. Sb. N.S. 48 (1938), 90-94.

[18] V.L. Śmulian, "Sur la dérivabilité de la norme dans l'espace de Banach", C.R. (Dokz.) Acad. Sci. URRS 27 (1940), 643-648.

[19] V.L. Smulian, "Sur la structure de la sphère unitaire dans l'espace de Banach", Rec. Math. Mat. Sb. N.S. (51) 9 (1941), 545-561.

[20] Francis Sullivan, "Some geometric properties of higher duals of Banach spaces", Proc. Conf. Radon-Nikodym Prop. Kent State (Preprint). 
[21] L. Tzafriri, "Reflexivity in Banach lattices and their subspaces", J. Functional Analysis 10 (1972), 1-18.

Institute of Mathematics,

Str. Academiei 14 ,

Bucuresti,

Romania. 